\title{
Performance Analysis for Visual Planetary Landing Navigation Using Optical Flow and DEM Matching
}

\author{
K. Janschek ${ }^{1}, \mathrm{~V}$. Tchernykh ${ }^{2}, \mathrm{M}$. Beck $^{3}$ \\ Technische Universität Dresden, Department Electrical Engineering \& Information Technology, \\ D-01062 Dresden, Germany
}

\begin{abstract}
Visual navigation for planetary landing vehicles shows many scientific and technical challenges due to inclined and rather high velocity approach trajectories, complex 3D environment and high computational requirements for real-time image processing. High relative navigation accuracy at landing site is required for obstacle avoidance and operational constraints. The current paper discusses detailed performance analysis results for a recently published concept of a visual navigation system, based on a mono camera as vision sensor and matching of the recovered and reference 3D models of the landing site. The recovered 3D models are being produced by real-time, instantaneous optical flow processing of the navigation camera images. An embedded optical correlator is introduced, which allows a robust and ultra high-speed optical flow processing under different and even unfavorable illumination conditions. The performance analysis is based on a detailed software simulation model of the visual navigation system, including the optical correlator as the key component for ultra-high speed image processing. The paper recalls the general structure of the navigation system and presents detailed end-to-end visual navigation performance results for a Mercury landing reference mission in terms of different visual navigation entry conditions, reference DEM resolution, navigation camera configuration and auxiliary sensor information.
\end{abstract}

\section{Introduction}

$\mathrm{F}$ uture Solar System Exploration missions will require the capability to perform precision autonomous guidance and navigation to the selected landing site. Pinpoint landing capability will allow to reach landing sites which may lie in areas containing hazardous terrain features (such as escarpments, craters, rocks or slopes) or to land accurately at select landing sites of high science value.

To make such a precision landing possible, a fast and accurate navigation system is required, capable of real time determination of the spacecraft position and attitude with respect to the planet surface during descent.

State of the art planetary landers are mainly navigated on the base of initial position knowledge at the start of descent phase by propagation of position data with the help of an inertial measurement unit. The initial lander position (before starting the deorbit burn) is usually determined by Earth-based observation with limited accuracy due to the large observation distance. The accuracy is further degrading during the inertial navigation phase due to error accumulation. As a result, the landing accuracy is considerably poor with the landing error about $30-300 \mathrm{~km}$ for planets with atmosphere and about $1-10 \mathrm{~km}$ for airless bodies ${ }^{1}$.

Much better accuracy, especially for the final part of the landing trajectory, can be obtained by visual navigation with respect to the surface. A number of concepts of visual navigation systems for planetary landing missions have been already developed and partially tested ${ }^{1-4}$. Their operation is generally based on the motion estimation by feature tracking and/or landmark matching. These approaches however have certain disadvantages. Navigation data determination by feature tracking is subject to error accumulation (results in accuracy degradation for the final part of the landing trajectory). Landmark matching requires the presence of specific features, suitable to be used as landmarks, within the field of view of navigation camera during the whole landing trajectory. Matching of 2D images of landmarks is also strongly affected by perspective distortions and changing illumination conditions.

${ }^{1}$ Full Professor, Managing Director, Institute of Automation, D-01062 Dresden, Germany, klaus.janschek@tudresden.de

${ }^{2}$ Senior Scientist, Institute of Automation, D-01062 Dresden, Germany, valerij.tchernykh@tu-dresden.de

${ }^{3}$ Scientific Staff, PhD candidate, Institute of Automation, D-01062 Dresden, Germany, martin.beck@tu-dresden.de 
The current paper discusses a recently introduced visual navigation concept ${ }^{5}$, which uses a correlation based optical flow approach for landing vehicle ego-motion estimation and 3D map generation. The demanding processing requirements for real-time optical flow computation can be met by an embedded optical correlator, which allows a robust and ultra high-speed optical flow processing under different and even unfavorable illumination conditions. This optical processor technology has been developed in the last years at the Institute of Automation, Technische Universität Dresden. First investigations considering realistic performance properties of the optical correlator hardware and dimensioning landing trajectory properties have proved the feasibility of the proposed concept ${ }^{5}$.

A more detailed performance analysis is given in the current paper in terms of different visual navigation entry conditions, reference DEM resolution, navigation camera configuration and auxiliary sensor information.

\section{Visual Navigation System Concept}

\section{A. General Concept}

Optical flow techniques allow to derive different types of visual navigation data (visual navigation observables), which can be used alternatively or in combination applying data fusion techniques in order to estimate motion parameters and 3D localization and attitude information relative to the surface. The fusion of the different visual navigation observables as well as with auxiliary navigation data (IMU, etc.) is performed in a high level navigation filter. In general the optical flow based navigation observables can be partitioned into the following three groups:

- egomotion observables: velocity, angular rate resp. position increment, attitude increment (visual odometry)

- 3D observables: local depth

- vehicle pose observables: position and attitude (=: pose), in general these are instantaneous pose estimates without time filtering.

The paper presents a basic concept, which derives all three types of visual navigation observables by instantaneous measurements of optical flow fields from a single navigation mono-camera (Fig. 1). It is assumed, that an onboard

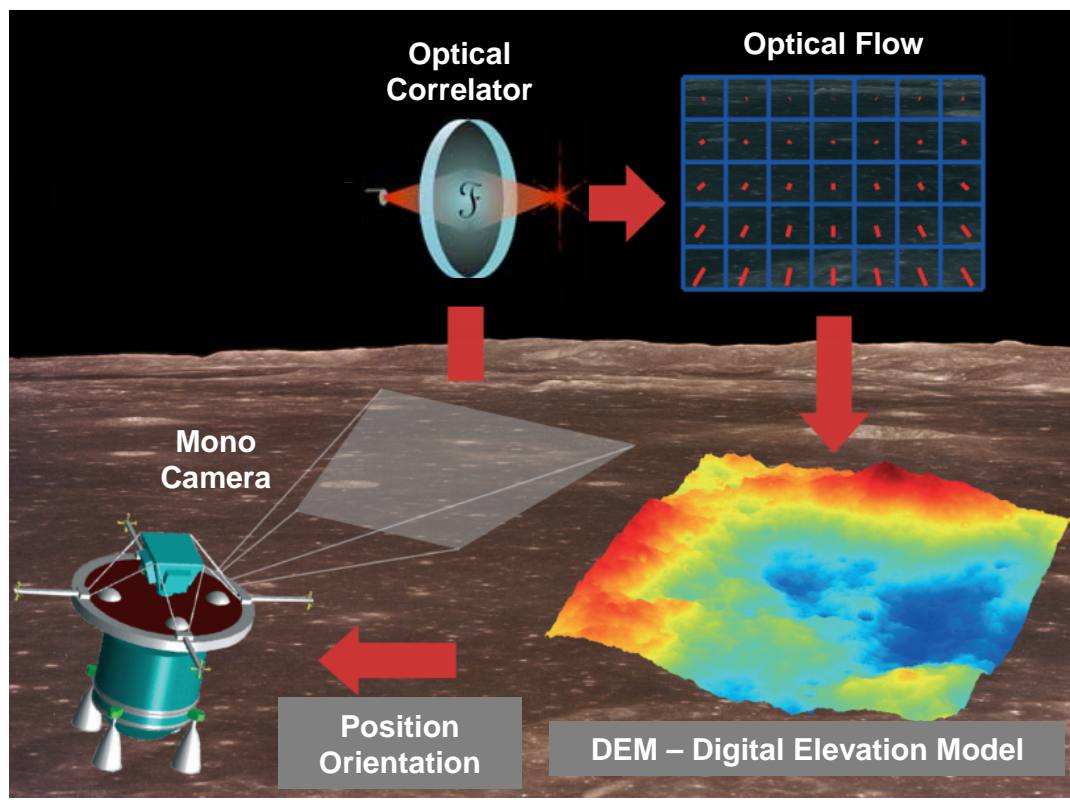

Figure 1. General concept of visual navigation system.

map of the landing area is available in terms of a reference Digital Elevation Model (DEM) and/or a set of reference images geometrically referenced to the planet surface.

To obtain the required accuracy and reliability of navigation data determination, the proposed concept is based on the matching of 3D models of the planet surface, produced from the optical flow field of the navigation monocamera. The instantaneous reconstructed 3D models of the visible surface are matched with a reference model of the 
landing site. Position and attitude of the lander with respect to the surface will be determined from the results of the matching. To reach the required real time performance together with high accuracy of 3D models, the optical flow fields will be determined by multipoint 2D correlation of sequential images with an onboard optical correlator (Fig. 2).

Matching of 3D models instead of 2D images offers several advantages, in particular it is not sensitive to perspective distortions and is therefore especially suitable for inclined descent trajectories. The method does not require any specific features/objects/landmarks on the planet surface and is not affected by illumination variations. The redundancy of matching the whole surface instead of the individual reference points ensures a high reliability of matching and a high accuracy of the obtained navigation data: generally, the errors of lander position determination are expected to be few times smaller, than the resolution of the reference model.

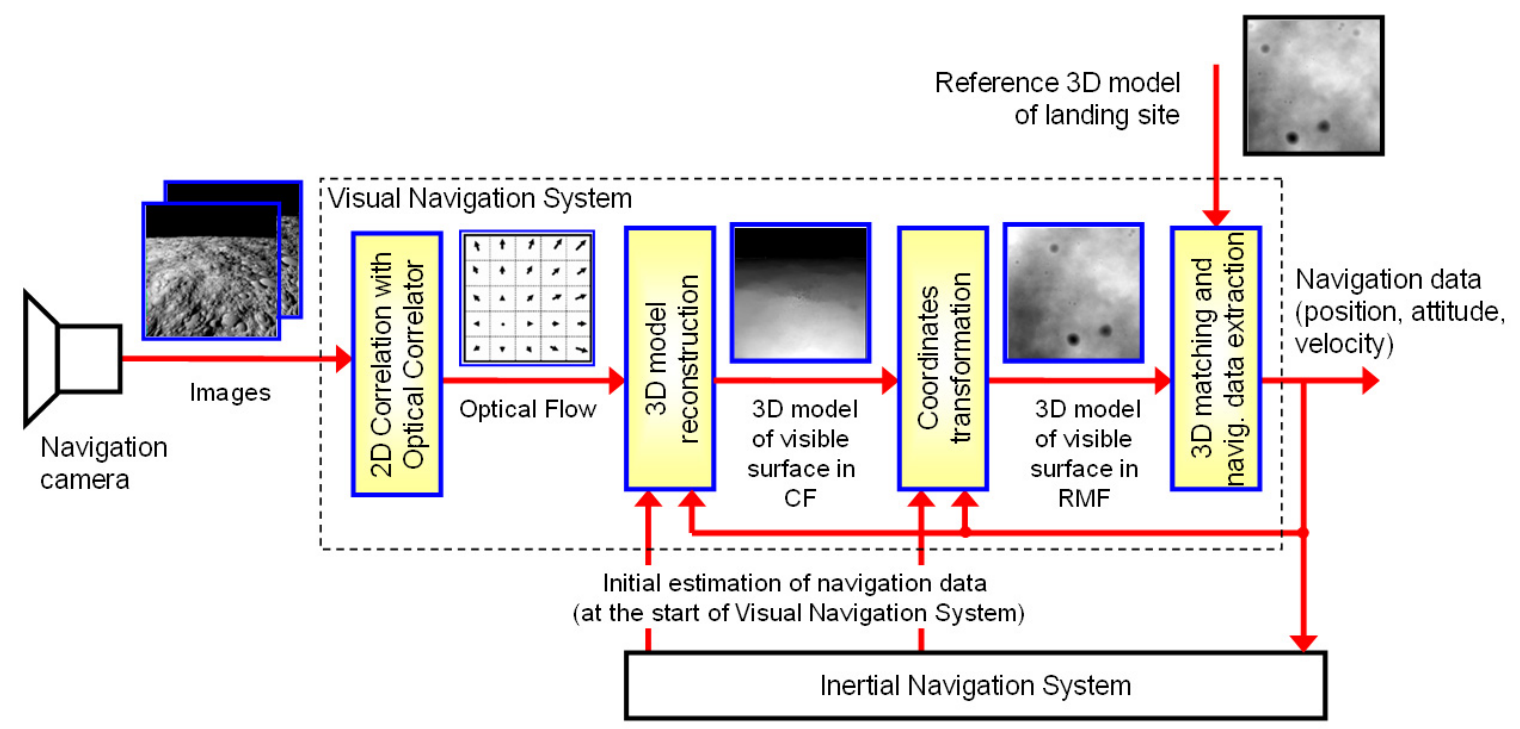

Figure 2. Visual navigation system functional architecture.

\section{B. Application Area}

The system is intended to produce high accuracy navigation data (position, attitude and velocity) in a planetfixed coordinate frame (PF) during descent of the planetary lander. The lander should be equipped with a planetoriented navigation camera (exact nadir orientation is not necessary; the camera can look forward-down, for example).

For optimal performance the system can work in parallel with an inertial navigation unit, which allows coarse orientation of the lander and provides initial rough estimats of navigation data at the initialization of the visual navigation phase. During operation, the system can also benefit from real time acceleration information.

A totally autonomous operation is possible in principle, at the cost of considerably long start-up time and some degradation of performances. In autonomous mode a certain degree of attitude stabilization is required prior to start of system operation (navigation camera should see the planet surface; rotation velocity of the spacecraft should be within $5 \ldots 25 \%$, depending on the mission parameters).

\section{Inputs Required}

The system determines the navigation data by processing of images of the planet surface derived from a navigation camera. Besides images, the following inputs are required:

1. Reference 3D model of the landing site in a planet-fixed frame (can be obtained from orbital observations prior to landing, e.g. by processing of stereo images or radar data)

2. Initial rough estimates of position, attitude and velocity (at the initialization of the visual navigation system)

3. Camera parameters 


\section{Optical Flow Determination}

\section{A. 2D Correlation based Optical Flow Computation}

The egomotion of a camera rigidly mounted on a vehicle is mapped into the motion of image pixels in the cameras focal plane. The resulting vector field of $2 \mathrm{D}$ image motion is commonly understood as image flow or optical flow ${ }^{6}$ and can be used efficiently for navigation purposes such as localization, mapping, obstacle avoidance or visual servoing. The big challenge for using the optical flow in real applications is its computability in terms of its density (sparse vs. dense optical flow), accuracy, robustness to dark and noisy images and its real-time determination.

In particular the navigation in unstructured environments with dark and noisy images due to small camera aperture or fast vehicle dynamics requires a robust determination of the apparent image motion. For practical image motion tracking applications the so called block or window based approach using area methods has proved to be very robust. The underlying methods exploit the temporal consistency over a series of images, i.e. they assume that the appearance of a small region in an image sequence changes little.

The classical and most widely used approach is the area correlation, applied originally for image registration ${ }^{7}$. Area correlation uses the fundamental property of the cross-correlation function of two images, which gives the location of the correlation peak directly proportional to the displacement vector of the original image shift. The optical flow matrix thus can be computed by segmentation of an image pair into fragments (windows) and computing of the mutual image shift vectors for each pair of corresponding fragments by 2D correlation (Figure 3).

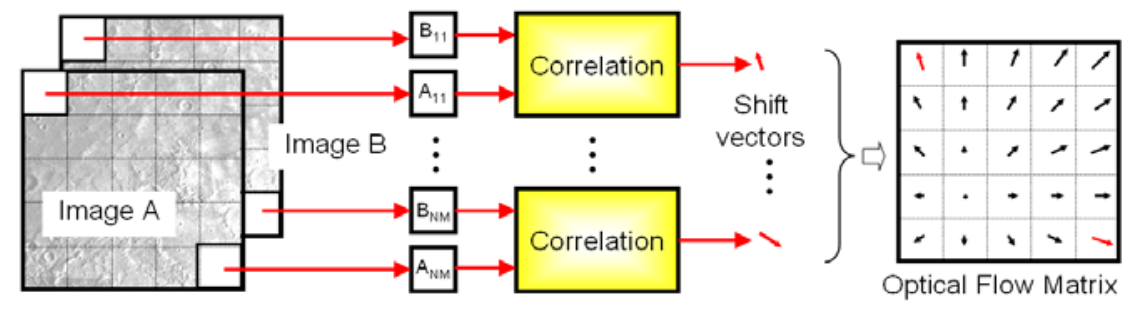

Figure 3. Principle of the correlation based optical flow determination.

The optical flow determination method, based on 2D correlation of the image fragments, offers a number of advantages:

- high subpixel accuracy (error of image shift determination can be within 0.1 pixel , $1 \sigma$ );

- low dependency on image texture properties (no specific texture features required);

- high robustness to image noise (suitable for short exposures and/or poor illumination conditions)

- direct determination of large (multi-pixel) image shifts (suitable for measuring fast image motion).

\section{B. Joint Transform Optical Correlation}

A very efficient method for 2D correlation requiring only double Fourier transform without phase information is given by the Joint Transform Correlation (JTC) principle . $^{8}$

The two images $f_{1}(x, y)$ and $f_{2}(x, y)$ to be compared are being combined to an overall image $I(x, y)$. A first Fourier transform results in the joint power spectrum $S(u, v)=F\{I(x, y)\}$. Its magnitude contains the spectrum $F(u, v)$ of the common image contents augmented by some periodic components which are originating from the spatial shift $\vec{G}$ of $f_{1}$ and $f_{2}$ in the overall image $I$. A second Fourier transform of the squared joint spectrum $J(u, v)=S(u, v)^{2}$ results in four correlation functions. The centred correlation function $C_{f f}(x, y)$ represents the autocorrelation function of each input image, whereas the two spatially shifted correlation functions $C_{f f}\left(x \pm G_{x}, y \pm G_{y}\right)$ represent the cross-correlation functions of the input images. The shift vector $\vec{G}$ contains both the technological shift according to the construction of the overall image $I(x, y)$ and the shift of the image contents according to the image motion. If the two input images $f_{1}$ and $f_{2}$ contain identical (but shifted) image contents, the cross- 
correlation peaks will be present and their mutual spatial shift $\vec{\Delta}=\vec{G}-(-\vec{G})$ allows determining the original image shift in a straightforward way.

This principle can be realized in hardware by a specific opto-electronic setup, named Joint Transform Optical Correlator (JTOC). The required 2D Fourier transforms are performed by means of diffraction-based phenomena, incorporating a so called optical Fourier processor ${ }^{9}$. A laser diode generates a diverging beam of coherent light which passes a single collimating lens focusing the light to infinity. The result is a beam of parallel light with plane wave fronts. The amplitude of the plane wave front is modulated by a transmissive spatial light modulator (SLM), a special transparent liquid crystal display. The SLM actually works as a diffraction brig and the resulting diffraction pattern can be made visible in the focal plane of a second lens (Fourier lens). Under certain geometric conditions the energy distribution of this pattern is equal to the squared Fourier transform (power spectrum) of the modulated wave front. The power spectrum can be read by a CCD or CMOS image sensor located in the focal plane of the Fourier lens of the optical Fourier processor. The position of the correlation peaks in the second power spectrum (correlation image) and the associated shift value can be measured with sub-pixel accuracy using standard algorithms for centre of mass calculation.

Optical processing thus allows unique real time processing performances of high frame rate video streams. TU Dresden has developed an embedded hardware model of a JTOC with a performance 60 correlations $/ \mathrm{sec}^{10}$. Due to special design solutions owned by TU Dresden, the devices are robust to mechanical loads and deformations ${ }^{11-13}$.

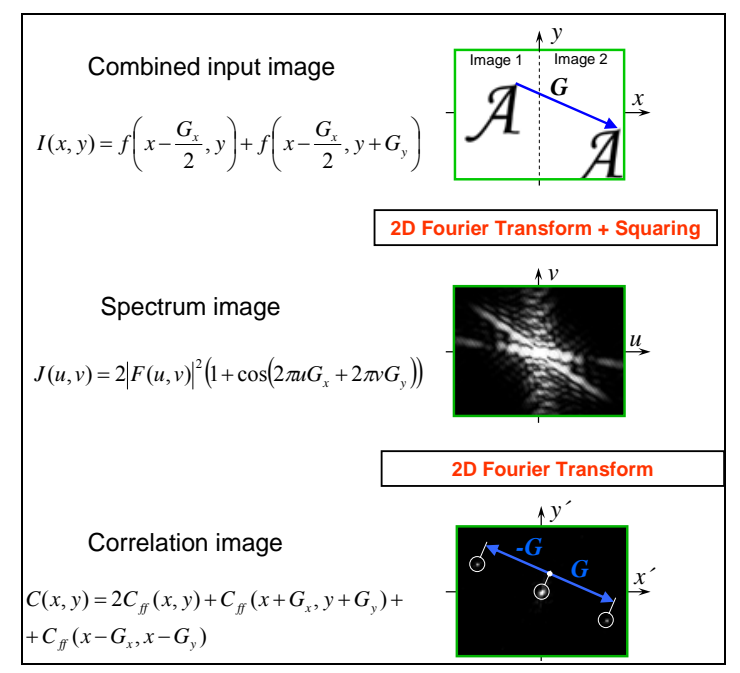

Figure 4. Principle of the joint transform correlation.

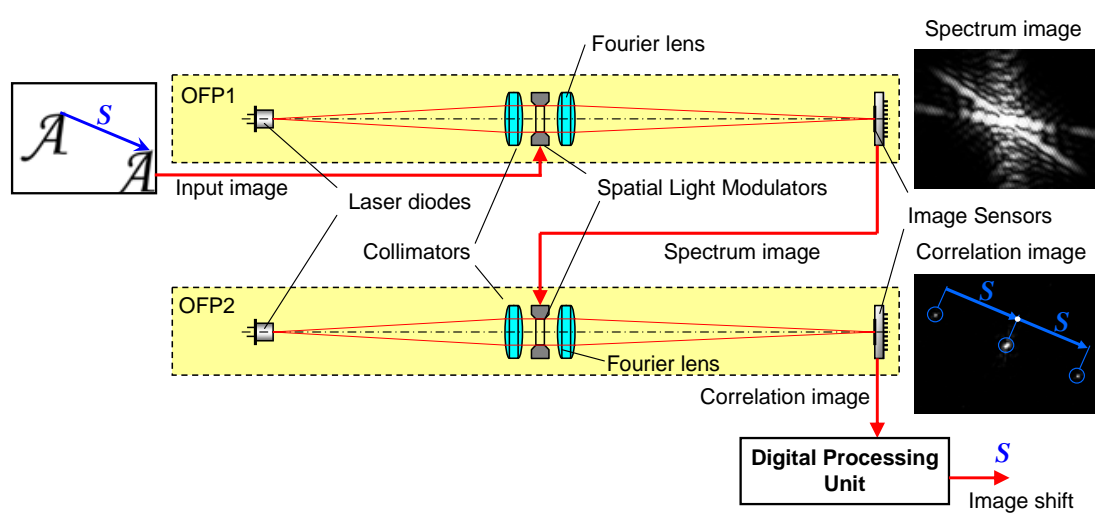

Figure 5. Principle of the Joint Transform Optical Correlator (JTOC). 


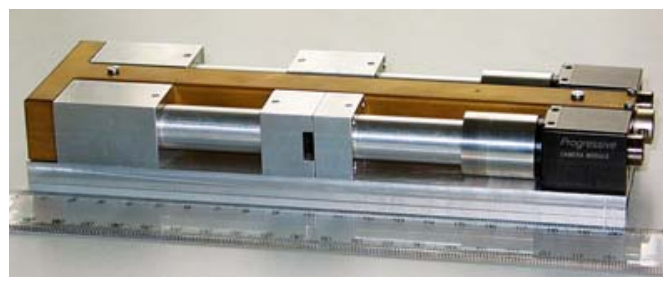

Figure 6. Hardware model of TU Dresden optical correlator (60 correlations/sec).

\section{Advanced Opto-Electronic Optical Flow Processor}

The maximum correlation speed (frames per second) is directly linked to the interface rates of the SLM and the spectrum image sensor. For highest performance rates the following optical correlator design based on advanced customized miniature optoelectronic components is proposed. A reflective Spatial Light Modulator (SLM) and a Spectrum/Correlation Image Sensor (SCIS) are configured in a folded optical system design on the base of small block of glass or optical plastic to reduce the overall processor size.

The operation differs slightly from the standard JTOC design in transmissive configuration. In the folded design the coherent light, emitted by a laser diode, reflects from the aluminized side of the block and illuminates the SLM surface via the embedded lens (can be formed as a spherical bulb on the surface of the block). The phase of the wave front reflected from the SLM, is modulated by the input image. It is focused by the same lens and forms (after intermediate reflection) the amplitude image of the Fourier spectrum of input image on the SCIS surface. After a second optical Fourier transform, the correlation image is obtained. The optical flow vector (equal to the shift between the correlated fragments) is calculated from the correlation peaks positions within the correlation image. This operation can be performed directly inside the SCIS chip. Using unpackaged optoelectronic components and Chip On Board (COB) mounting technology, the whole optical system can be realized within the volume of $6 \times 15 \mathrm{x}$ $20 \mathrm{~mm}$.

The image segmentation and the interfacing with the navigation camera and the further optical flow processing will be performed within a FPGA chip (compact BGA package) on a single printed board in the aluminum housing. The dimensions of correlated fragments determine the accuracy and reliability of correlation. Larger window size improves the reliability of the optical flow determination in poorly textured image areas and reduces the errors of the obtained OF vectors. At the same time, increasing the correlated fragments size will smooth the obtained OF field, it will suppress small details and it will produce additional errors in areas with large variations of local depth.

A detailed sensitivity analysis within a window range from $8 \times 8$ to $64 \times 64$ pixels as shown an optimal window size of $24 \times 24$ pixels at a distance of 12 pixels (1/2 of correlated fragment size), making the best compromise between the accuracy/reliability of correlation and preservation of small details of the underlying 3D scene ${ }^{14}$.

Expected performances of the optical flow processor have been estimated on the base of the conceptual design of the processor and results of simulation experiments, taking into account also the test results of the existing hardware models of the optical correlator made within previous studies ${ }^{10-11}$.

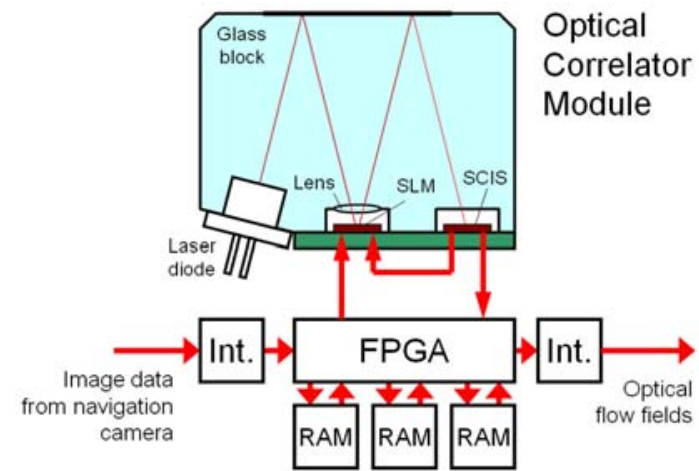

Figure 7. Advanced opto-electronic optical flow processor with optical correlator as core component. 
Table 1. Expected performances of advanced opto-electronic optical flow processor.

\begin{tabular}{|l|l|}
\hline Input & Images $1024 \times 1024$ pixels \\
\hline Output & optical-flow fields \\
\hline Optical-flow resolution & $70 \times 70=4900$ vectors/field \\
\hline OF fields rate & 10 fields $/ \mathrm{s}$ \\
\hline Processing delay & One frame $(0.1 \mathrm{~s})$ \\
\hline Inner correlations rate & 50000 correlations/s \\
\hline OF vectors determination errors & $\sigma=0.1 \ldots 0.25$ pixels \\
\hline Optical correlator dimensions (w/o housing) & $25 \times 20 \times 12 \mathrm{~mm}$ \\
\hline Optical correlator mass (w/o housing) & within $10 \mathrm{~g}$ \\
\hline Power consumption (optical correlator only) & within $1 \mathrm{~W}$ \\
\hline
\end{tabular}

\section{Visual Navigation Functions}

\section{A. 3D Model Reconstruction}

The bilinear optical flow egomotion equation ${ }^{1}$ relates the superposed rotational and translational optical flow to camera (vehicle) angular rate and translational velocity scaled by the local depth of the scene. Due to the bilinear characteristic only the velocity direction (not the magnitude) can be retrieved from pure optical flow field. Several approaches are known in literature to solve for the egomotion observables velocity direction and angular rate as well as for the local depths. The approach followed for the current investigations uses the discrete equivalent of the continuous optical flow egomotion equation (Fig. 8):

$$
R^{C F_{2} C F_{1}}\left(\begin{array}{l}
v_{1, x} \\
v_{1, y} \\
1
\end{array}\right) z_{1}-\left(\begin{array}{l}
v_{2, x} \\
v_{2, y} \\
1
\end{array}\right) z_{2}=\left(\begin{array}{l}
t_{x 2} \\
t_{y 2} \\
t_{z 2}
\end{array}\right)
$$

with

- $\quad x_{1}, y_{1}$ and $z_{1}$ are the object (surface point) coordinates in camera-fixed coordinate frame at the moment of the first image acquisition $\mathrm{CF}_{1}$ ( $\mathrm{z}$ axis coincides with optical axis);

- $\quad x_{2}, y_{2}$ and $z_{2}$ are the object (surface point) coordinates in camera-fixed coordinate frame at the moment of the second image acquisition $\mathrm{CF}_{2}$.

- $v_{1, x}=x_{1} / z_{1}, v_{1, y}=y_{1} / z_{1}, v_{2, x}=x_{2} / z_{2}$ and $v_{2, y}=y_{2} / z_{2}$ are the coordinates of the object (surface) image points measured in the focal plane of navigation camera (measured optical flow $=v_{2}-v_{1}$ );

- $R^{C F_{1}, C F_{2}}$ is the rotation matrix which aligns the attitudes of coordinate frames $\mathrm{CF}_{1}$ and $\mathrm{CF}_{2}$; the matrix is determined from the optical flow;

- $\vec{t}$ is the vector of camera motion in $\mathrm{CF}_{2}$ between the moments of images acquisition; direction of $\vec{t}$ is determined from optical flow, magnitude is estimated on base of previous measurements or data from other navigation aids.

The 3D surface model is produced from the matrix of optical flow vectors (raw optical flow). Each optical flow vector, determined from two sequential camera images, represents the motion of the corresponding object (surface point) projection in the focal plane of the camera during the time interval between the images exposures.

The $3 \mathrm{D}$ position of each surface point with respect to the camera is determined by solving Equ. (1) for a whole set of surface points coordinates, determined from all optical flow vectors. This forms the 3D model (local distance map, local depth map) of the visible surface in a camera-fixed coordinate frame CF. 


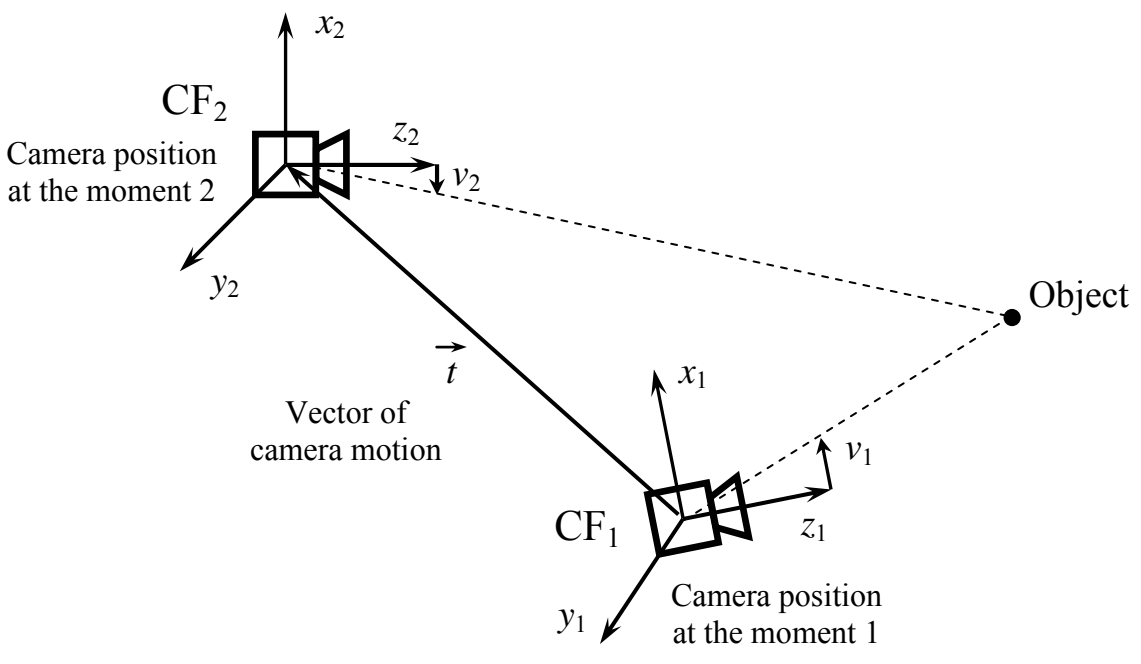

Figure 8. Camera motion with respect to the observed object.

\section{B. 3D Model Matching and Navigation Data Extraction}

To determine the actual position, attitude and velocity of the lander, the optical flow-derived 3D model of the visible surface is matched with the reference model of the landing site. For 3D-matching, the local depth map (reconstructed from optical flow), which is given in the camera-fixed coordinate frame CF, must be represented in the same coordinate frame as the reference model. This is done by a coordinate transformation, using the estimated (propagated) position $r_{E s t}$, attitude $R_{E s t}$ and velocity $v_{E s t}$ of the lander in a reference model-fixed frame (RMF) from the previous matching step or from the navigation filter.

With ideal (accurate) estimates, the reconstructed model in RMF should exactly coincide with the reference model. In the real case, the estimation errors result in some misalignment of the models. (Fig. 9).

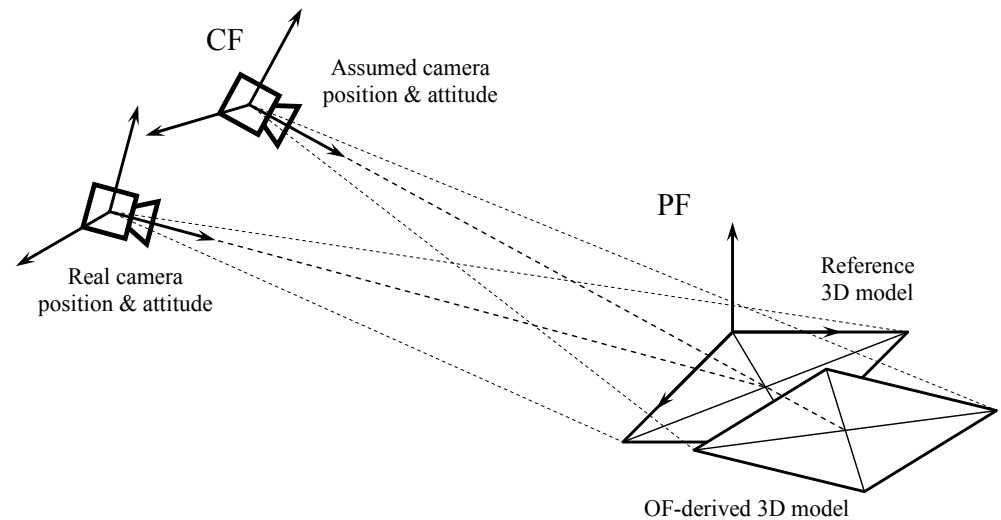

Figure 9. Misalignment of the optical flow-derived and reference DEM models.

Matching of the models is understood as finding the geometrical transformation, which aligns the reconstructed and reference surfaces. From the parameters of the matching transformation the errors of navigation data estimation can be determined. With these estimation errors known, an updated position, attitude and velocity estimate of the lander can be calculated.

It should be noted, that this calculation is done instantaneously, whenever a new image from the navigation camera is available. Thus an instantaneous navigation solution, i.e. instantaneous navigation observables of the lander pose and velocity, can be provided. This data can be smoothed and fused within the navigation filter as well used for integrity management with other navigation aids. Moreover these instantaneous estimates can be obtained 
completely autonomous, i.e. with sole visual information and without additional sensors. Nevertheless any auxiliary navigation data, e.g. IMU measurements, will simplify and improve robustness and accuracy (see simulation results in section VI).

\section{Simulation Experiment Description}

To prove the feasibility of the proposed visual navigation concept and to estimate the expected navigation performances, a detailed software model of proposed visual navigation system has been developed and an open-loop simulation of navigation data determination has been performed.

The simulation experiments have been performed considering a reference mission scenario, based on the Mercury lander concept (originally planned as a part of Bepi Colombo mission). According to the mission scenario, the visual guidance phase starts at an altitude of $8.46 \mathrm{~km}$ (ground distance to landing site $20.1 \mathrm{~km}$, velocity $746 \mathrm{~m} / \mathrm{s}$ ) and ends at an altitude $142 \mathrm{~m}$ (ground distance $40 \mathrm{~m}$, velocity $36 \mathrm{~m} / \mathrm{s}$ ). The reference mission scenario also included the landing trajectory (time dependence of lander position and attitude during descent). The nominal camera orientation is a forward looking camera with a mean inclination of $\delta=32^{\circ}$ (see also Fig. 24).

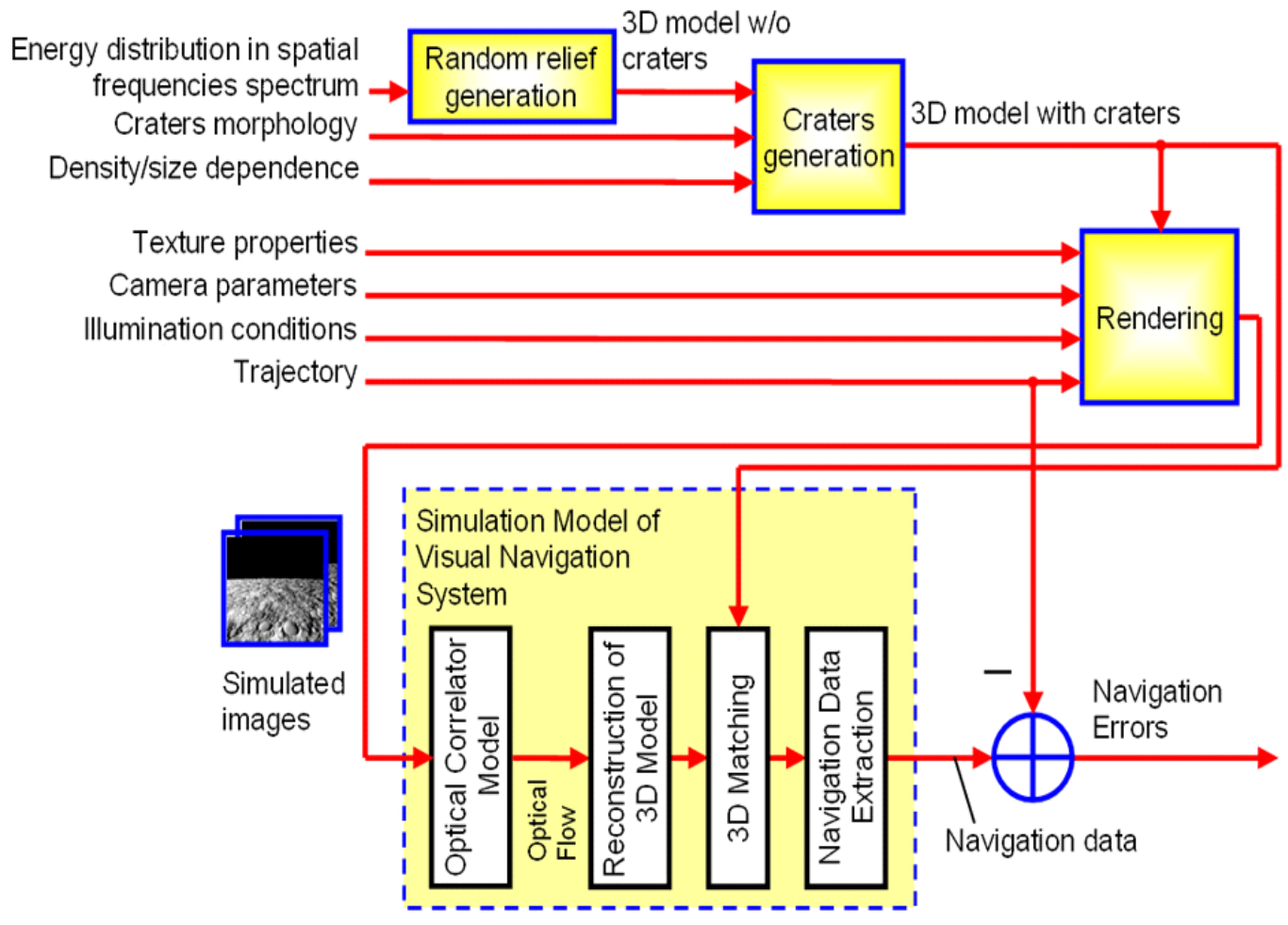

Figure 10. Simulation experiment (main operations).

The following main operations have been performed in the simulation experiments (Fig. 10):

$3 D$ model generation of the landing site has been performed in a form of a Digital Elevation Model (DEM). Such model can be represented by a 2D pseudo image, with brightness of each pixel corresponding to the local height over the base plane. The model has been generated on base of a random relief field. To produce the naturally looking landscape, a radial distribution of the energy in the spatial frequencies spectrum of the generated relief has been adjusted to match with that of the typical relief of the target planet. Craters have been added to the generated relief considering typical density/size dependence and morphology. Overlapping and partial destruction of old craters by younger ones have been simulated. To provide the required resolution for the whole descent trajectory, the 
model has been produced as a combination of 12 embedded layers, each containing 4096x4096 pixels. Figure 11 shows one of these layers.

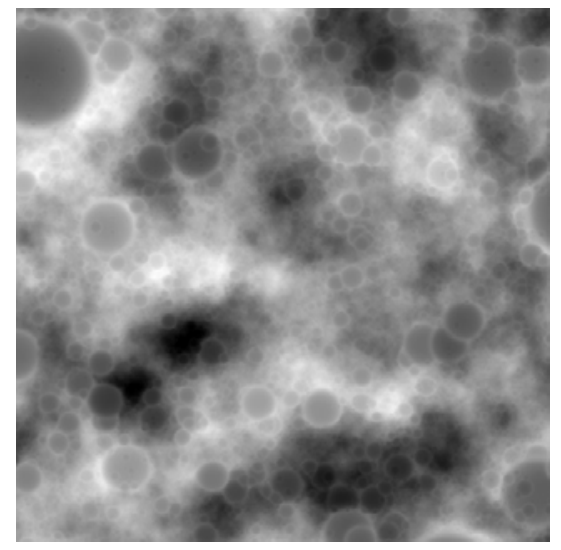

Figure 11. Simulated DEM of landing site (one layer).

The synthetic image generation has been performed using a 3D model of the landing site. Rendering of simulated images have been made using standard ray tracing software (Pov-Ray, version 3.6), taking into account the lander trajectory and camera parameters. Resolution of the rendered images was set to 4096x4096 pixels, what is four times higher, then the assumed resolution of the navigation camera (1024x1024 pixels). A higher resolution of the rendered images has been used later for MTF simulation of the optics and image sensor.

Rendering has been performed assuming uniform texture of planet surface with constant albedo and Lambertian light scattering. The surface has been illuminated by a point light source, positioned at infinite distance (parallel light) with elevation $30^{\circ}$ over horizon and azimuth $-110^{\circ}$ with respect to the flight direction.

MTF of the optics ( 0.6 at Nyquist frequency) has been applied in the Fourier plane. For this purpose a 2D Fourier transform of the image (4096x4096 pixels) has been performed, the obtained spectrum was multiplied by the desired MTF envelope and reversely transformed to obtain the filtered image. The MTF of the image sensor has been simulated by down sampling (binning) of the large rendered image. Each pixel of the down sampled image (1024x1024 pixels) has been produced by averaging of the corresponding group of $4 \times 4$ pixels of the large (4096x4096 pixels) image. Photonic noise has been simulated by adding the random noise with standard deviation equal to square root of pixel brightness, measured in electrons (average brightness of the image has been assumed to be 13000 electrons). Readout noise has been simulated by adding the random noise with $\sigma=40$ electrons. Pixel response non-uniformity has been simulated by multiplication of the pixel brightness by 1 plus random value with $\sigma_{\mathrm{PRNU}}=2 \%$. Discretization has been simulated by rounding of the pixel brightness to 10 bits. Figure 12 shows an example of the synthetic navigation camera image.

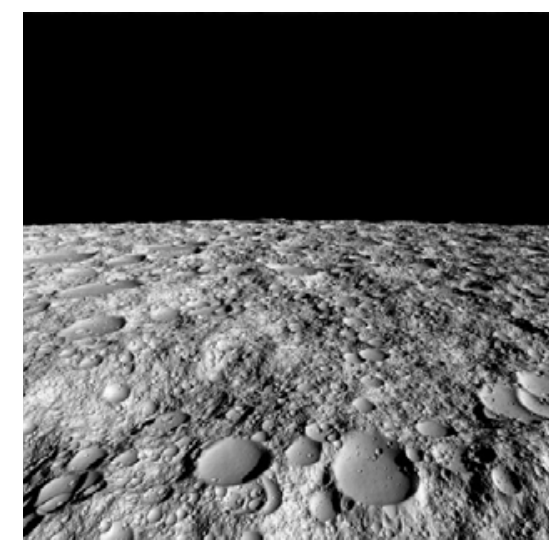

Figure 12. Example of synthetic image from navigation camera. 
Optical Flow determination has been performed with a detailed simulation model of the optical correlator. The correlator simulation model processes pairs of simulated navigation camera images and produces the optical flow fields (raw optical flow matrices) with required density. Image processing algorithms simulate the operation of the planned hardware realization of the optical correlator (optical diffraction effects, dynamic range limitation and squaring of the output images by image sensor, scaling of the output images according to focal length value, etc.). Figure 13 shows an example of the optical flow field (magnitude of the optical flow vectors is coded by brightness, direction - by color).

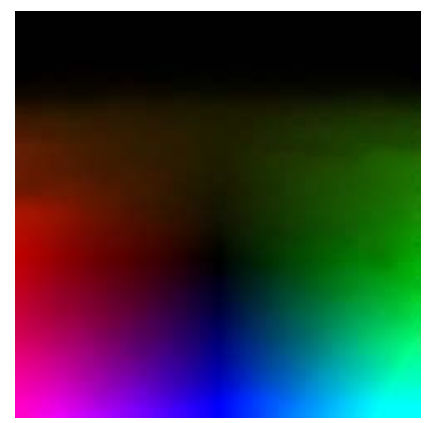

Figure 13. Optical flow field example (magnitude of optical flow vectors coded by brightness, direction by color).

Reconstruction of $3 D$ models of the visible surface, 3D-matching with the reference model and extraction of navigation data have been included in the software model of visual navigation system.

Simulation of errors of initial estimation of navigation data has been performed by adding the error values to the reference trajectory data and using the obtained sums as the initial estimates of position, attitude and velocity.

An example for the DEM derived from optical flow processing of the navigation camera images is shown in Fig. 14 (left). It has to be matched with the reference model as given in Fig. 14 (right).

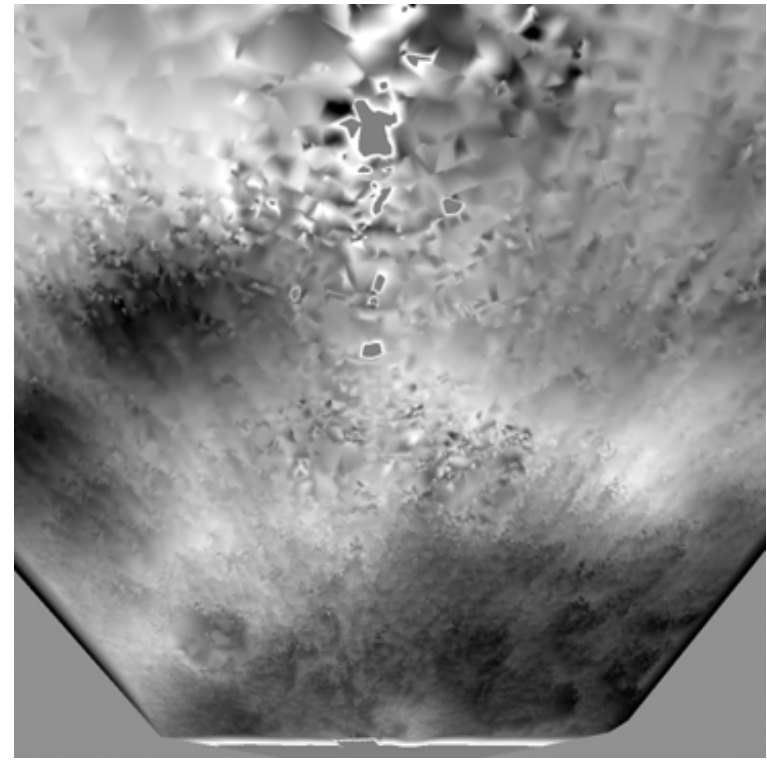

OF derived DEM

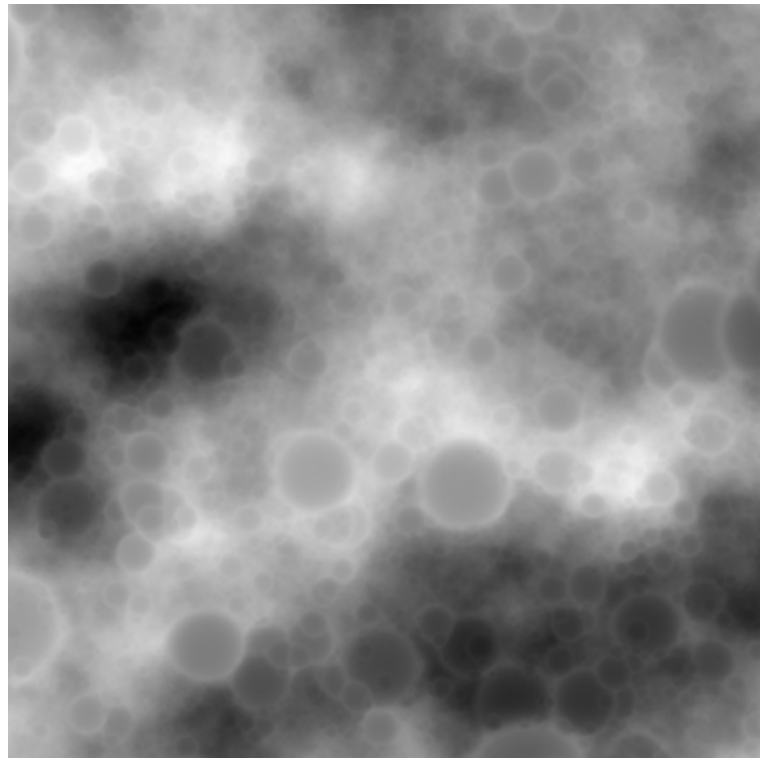

Reference DEM

Figure 14. Example of the OF-derived and reference DEMs of planet surface. 


\section{Performance Evaluation}

\section{A. Time Behaviour of Navigation Instantaneous Estimation Errors}

Figures 15 to 17 show the typical error time history of navigation instantaneous estimates, obtained as a result of simulation tests performed for the visual guidance phase of the landing trajectory. At initialization, the visual navigation system has been provided with the erroneous initial estimates of lander position, attitude and velocity, obtained as a sum of correct data from the reference trajectory and basic initial estimation errors: position $-500 \mathrm{~m}$ on each axis ( $866 \mathrm{~m}$ deviation from correct position $-4 \%$ of distance to landing site); attitude $-1.4^{\circ}$ for each angle; velocity $-20 \mathrm{~m} / \mathrm{s}$ on each axis ( $35 \mathrm{~m} / \mathrm{s}$ or $4.6 \%$ deviation from correct velocity). Full autonomous visual navigation has been simulated, i.e. beside camera images no other external sensor information was used. Position and velocity errors are measured w.r.t. planet fixed frame $(y-$ parallel to $\mathrm{S} / \mathrm{C}$ trajectory projected on the ground, $z$ - towards sky, $x$ - perpendicular to $y$ and $z$ ), attitude errors are measured w.r.t. camera fixed frame ( $x$ - to right, $y-$ down, $z-$ line of sight).

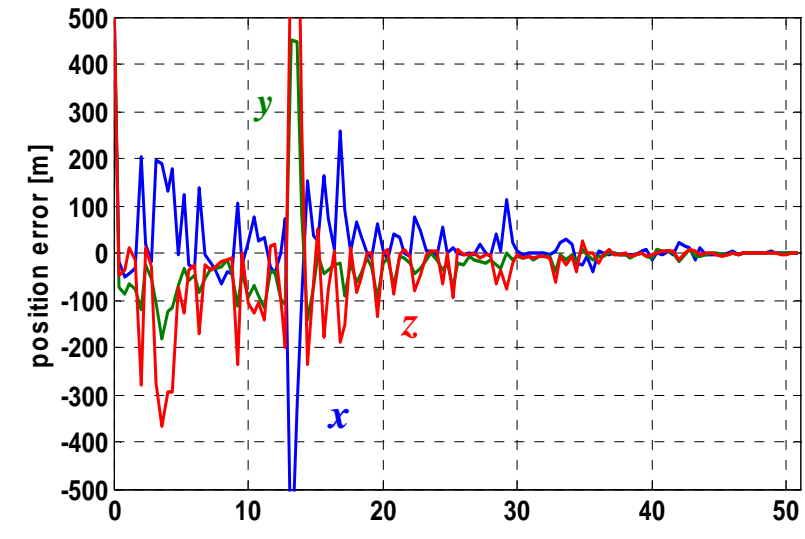

Figure 15. Position determination errors autonomous system.

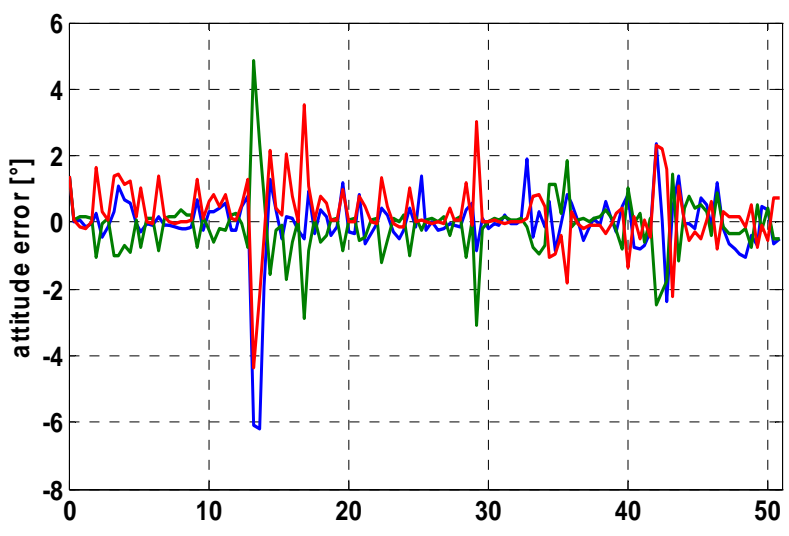

Figure 16. Attitude determination errors autonomous system.

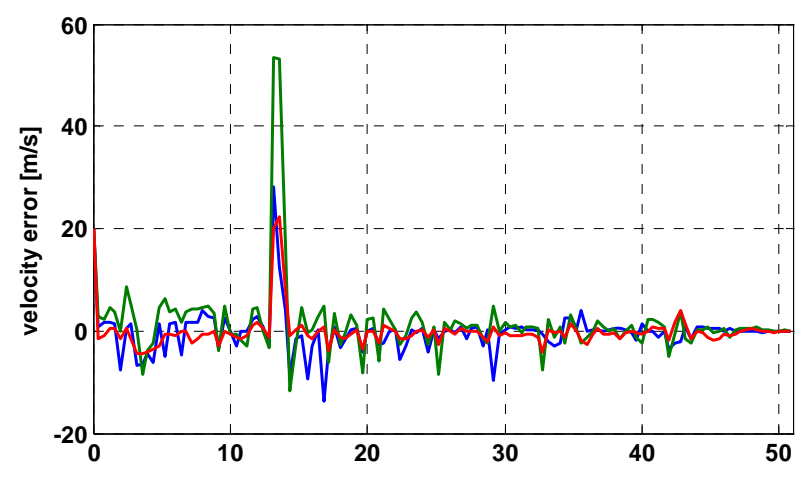

Figure 17. Velocity determination errors autonomous system.

The graphics show a fast compensation of the initial estimation error and proves the robustness of 3D model matching. The accuracy of position and velocity determination improves, as the lander approaches the landing point. The residual error of position determination at the end of visual guidance phase was $2.1 \mathrm{~m}$, velocity error $-0.49 \mathrm{~m} / \mathrm{s}$. The accuracy of attitude determination showed practically no dependence on the distance to the landing point. RMS attitude error during visual guidance phase has been determined to be within 1.6 degrees for the autonomous system. 


\section{B. Sensitivity to Initial Estimation Errors}

Figures 18 to 20 illustrate the effect of errors of initial position, attitude and velocity information on the navigation errors for the autonomous system (root sum square on all axes - only results of first 9 measurements are shown). The first experiment (Fig. 18) has been performed with initial position estimation error changed from 700 to $4000 \mathrm{~m}$ on each axis (attitude and velocity errors were constant and equal to the basic values, attitude errors $-1.4^{\circ}$ for each angle, velocity errors $-20 \mathrm{~m} / \mathrm{s}$ on each axis). The second experiment (Fig. 19) shows the results for initial attitude estimation error varying from $2^{\circ}$ to $11^{\circ}$ for each axis (position errors $-500 \mathrm{~m}$, velocity $-20 \mathrm{~m} / \mathrm{s}$ on each axis). The third experiment (Fig. 20) shows the results for initial with velocity estimation errors varying from 56 to $280 \mathrm{~m} / \mathrm{s}$ on each axis (position errors $-500 \mathrm{~m}$ on each axis, attitude errors $-1.4^{\circ}$ for each axis).
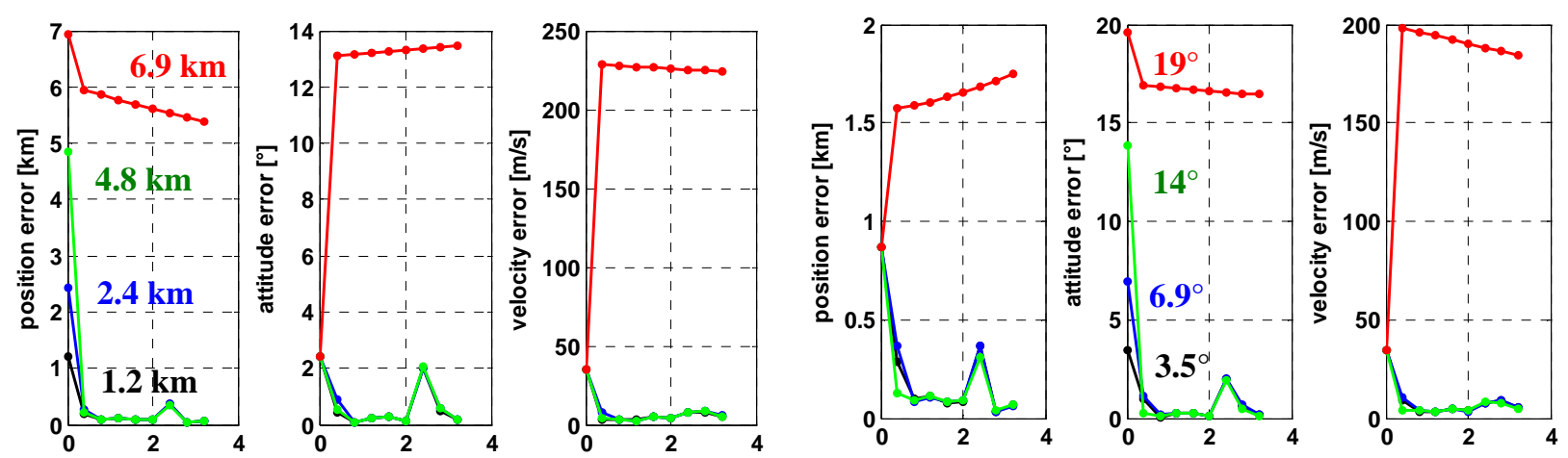

Figure 18. Effect of initial position estimation error - autonomous system.
Figure 19. Effect of initial attitude estimation error - autonomous system.
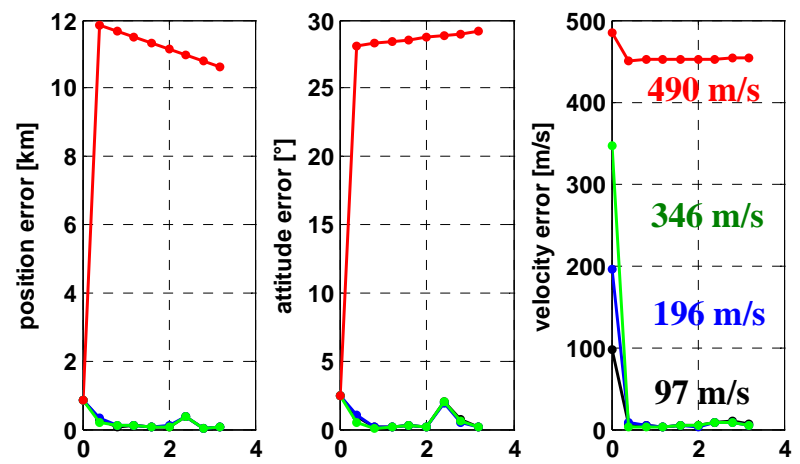

Figure 20. Effect of initial velocity estimation error - autonomous system.

As a result of the simulation studies a good estimation convergence can be expected, if the initial estimation errors are within certain limits and the effect of initial errors was practically compensated after 5 to 6 measurements. Acceptable limits of initial estimation errors (root mean sum on all axes) are given as:

- position estimation error - more, than $5500 \mathrm{~m}$ ( $25 \%$ of the initial distance to landing site);

- attitude estimation error - up to 7 degrees;

- velocity estimation error - more, then $150 \mathrm{~m} / \mathrm{s}$ ( $20 \%$ of initial velocity). 


\section{Sensitivity of Reference DEM Resolution}

Figures 21 to 23 show the dependency of navigation errors on the resolution of the reference DEM $(1 \mathrm{~m}, 4 \mathrm{~m}, 16 \mathrm{~m}$ and $64 \mathrm{~m}$ ). In this experiment, the egomotion estimates of the lander were taken from an external source.

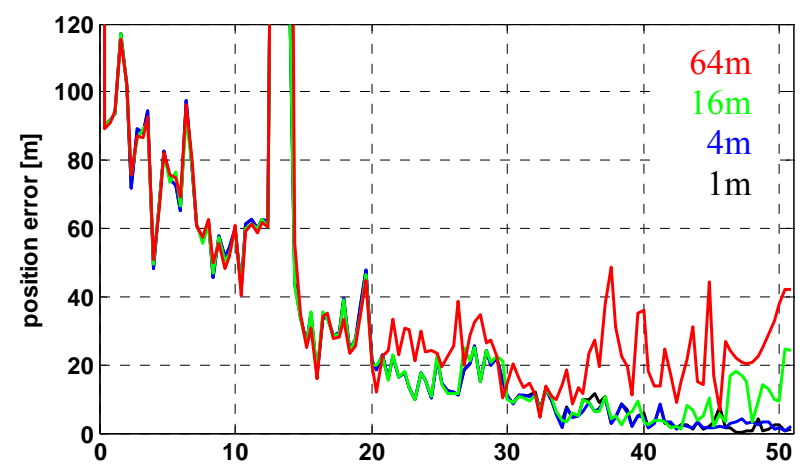

Figure 21. Position determination error - egomotion from external source.

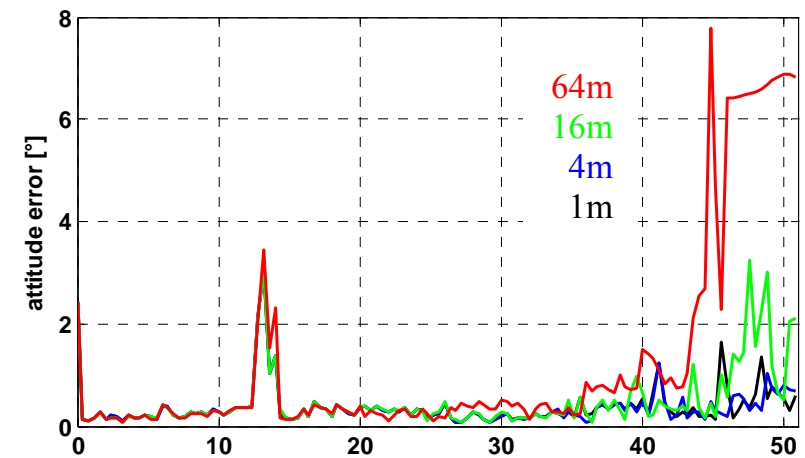

Figure 22. Attitude determination error - egomotion from external source.

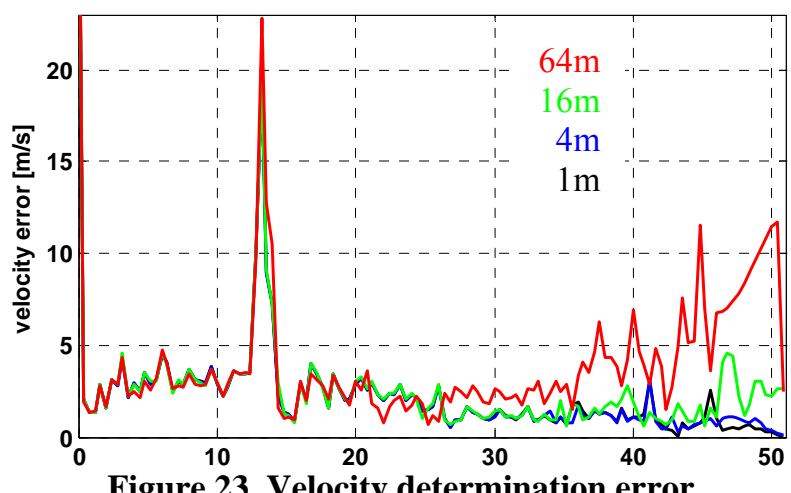

Figure 23. Velocity determination error - egomotion from external source.

The acceptable reference DEM resolution has found to be about 1/50 of navigation camera ground resolution for safe navigation. Even in this case the navigation accuracy (position determination error) is significantly better than the reference DEM resolution. 


\section{Sensitivity to Camera Orientation}

Further simulations have been performed to determine the dependency of navigation accuracy on camera orientation. Two configurations have been compared (Fig. 24):

1. forward looking camera with a mean inclination of $\delta=32^{\circ}$ (nominal configuration)

2. nadir looking camera with a mean inclination of $\delta=78^{\circ}$ (alternate configuration).

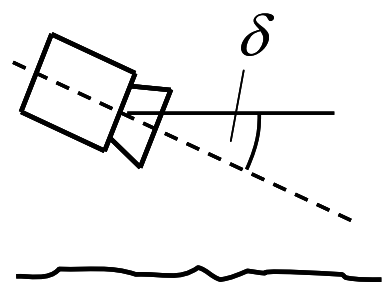

Figure 24.

Camera orientation

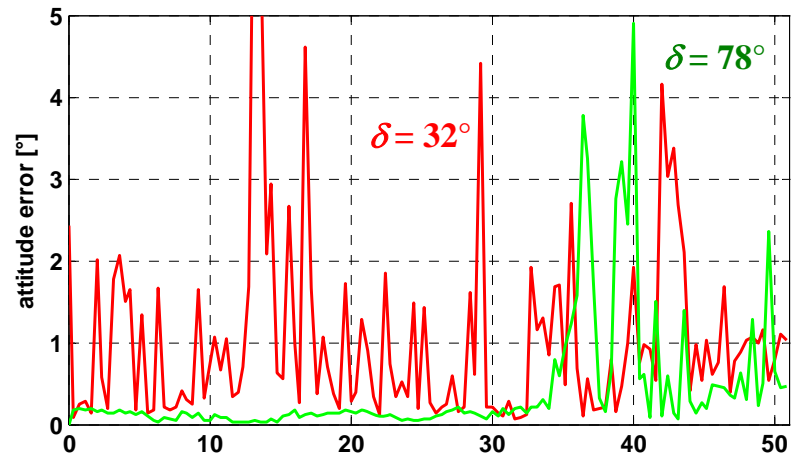

Figure 26. Attitude determination error autonomous system.

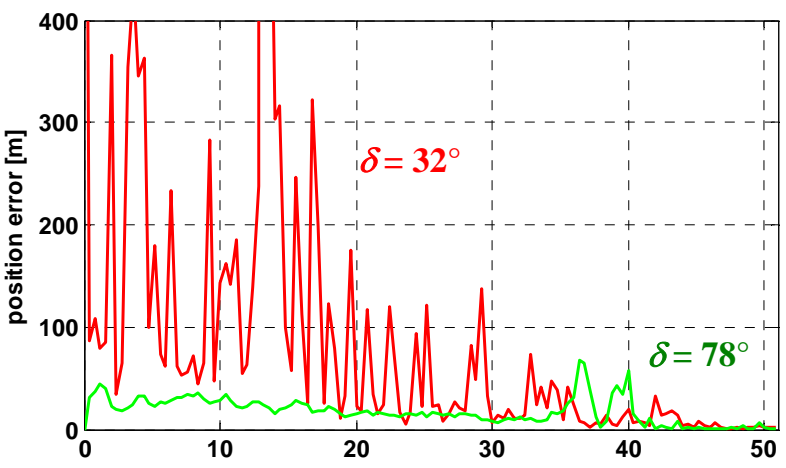

Figure 25. Position determination error autonomous system.

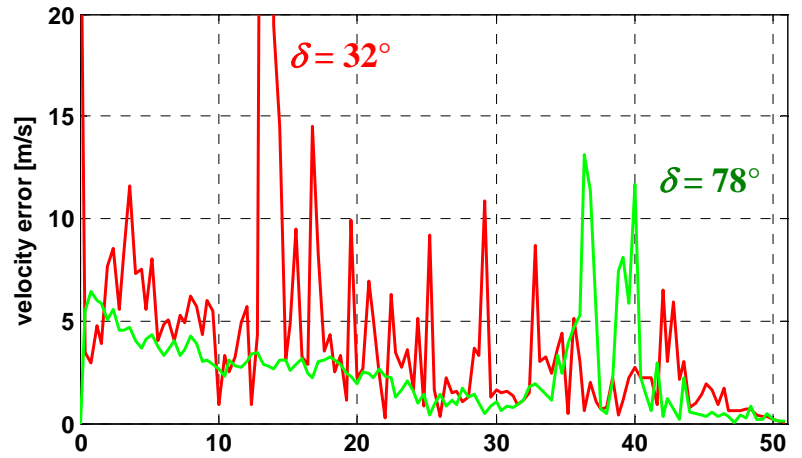

Figure 27. Velocity determination error autonomous system.

Figures 25 to 27 show much smaller navigation errors for the nadir looking camera. The advantage of this configuration is that no sky is visible where no navigation information is present. The lengths of the optical flow vectors are more or less constant over the entire image and image distortions during flight are significantly smaller than for the forward looking camera. This means the optical flow field contains much more valid vectors with improved accuracy. Consequently the vehicle egomotion determination and 3D surface model reconstruction will be more accurate. Additionally the 3D model matching benefits from the constant navigation camera ground resolution over the entire image, the full reconstructed DEM can be used for matching. 


\section{E. Effect of Auxiliary Sensor Information}

Another simulation experiment compares autonomous versus aided visual navigation. As expected, there is a considerable gain in navigation accuracy, when auxiliary navigation information is available and fused with the instantaneous visual navigation estimates. Figures 28 to 30 show the results for the autonomous system (red) and a system with inertial measurement unit (green). In the latter case the egomotion problem can be solved easily using the inertial measurement data. The optical flow will be used only for 3D model reconstruction.

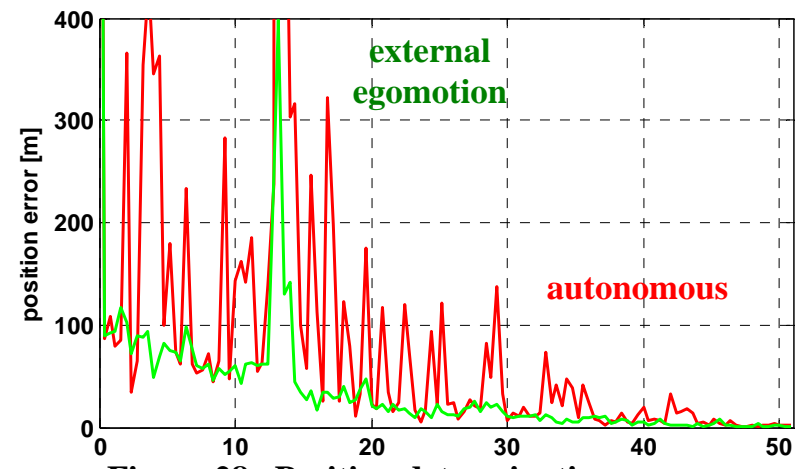

Figure 28. Position determination error.

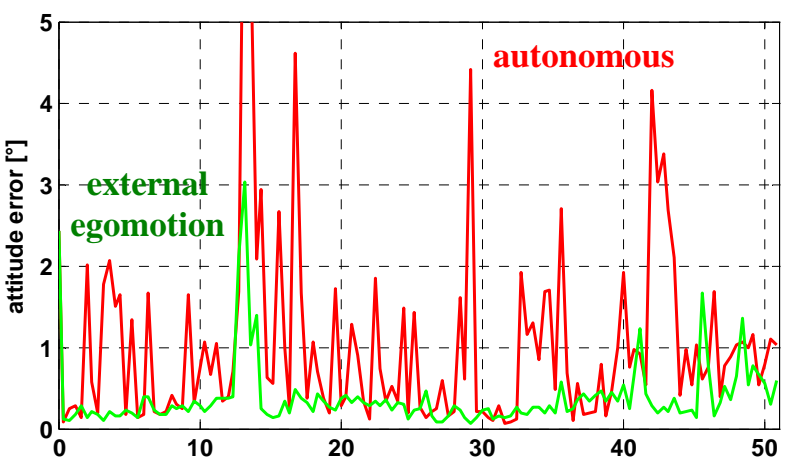

Figure 29. Attitude determination error.

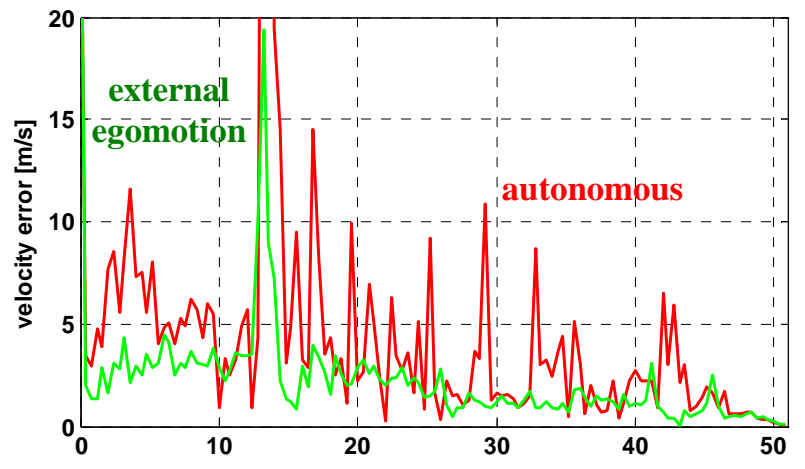

Figure 30. Velocity determination error.

\section{Conclusion}

A detailed performance analysis for a visual navigation concept for pin-point landing of a planetary lander has been presented, based on real time processing of optical flow data derived from a navigation mono-camera and an optical correlator. The correlation based optical flow computation shows to be suitable in particular for highly inclined landing trajectories and varying illumination conditions.

The practical realization of the proposed navigation system will allow to perform precision autonomous guidance and navigation to the selected landing site and to reach the locations of high scientific value also in areas containing hazardous terrain features.

The feasibility of proposed navigation system concept has been proved in open-loop simulation experiments, considering a Mercury landing scenario as a reference mission. The navigation errors in fully autonomous visual navigation at the end of simulated visual guidance phase were $1.6 \mathrm{~m}$ for position error, $0.2^{\circ}$ (one sigma) for attitude error and $0.15 \mathrm{~m} / \mathrm{s}$ for velocity error.

\section{Acknowledgments}

This research was partially supported by ESA/ESTEC under Contract No 18692/04/NL/MU. 


\section{References}

\footnotetext{
${ }^{1}$ Montgomery J. F., “Autonomous Vision Guided Safe and Precise Landing," - presentation at Automated Reasoning PI Workshop, September 2002

${ }^{2}$ Roumeliotis S.I., Johnson A.E., Montgomery J.F., “Augmenting inertial navigation with image-based motion estimation”, Proceedings of the ICRA'02, IEEE International Conference on Robotics and Automation, Vol. 4, pp. 4326 -4333, 2002.
}

3 Johnson A. E., Cheng Y., and Matthies L. H., "Machine vision for autonomous small body navigation," Aerospace Conference Proceedings, 2000 IEEE, Volume: 7, 18-25 March, pp. 661 -671 vol.7, 2000.

${ }^{4}$ Johnson A. E. and Matthies L. H., "Precise image-based motion estimation for autonomous small body exploration," in Proeedings of the.5th Int'l Symp. on Artificial Intelligence, Robotics and Automation in Space, Noordwijk, The Netherlands, June 1-3 1999, pp. 627-634.

${ }^{5}$ Janschek,K., Tchernykh, V., Beck, M., “An Optical Flow Approach for Precise Visual Navigation of a Planetary Lander”, in Proceedings of the 6th International ESA Conference on Guidance, Navigation and Control Systems, 17 - 20 October 2005 , Loutraki, Greece, ESA SP-606, January 2006.

${ }^{6}$ Horn, B.K.P., Schunck, B.G., “Determining Optical Flow”, Artificial Intelligence 17 (1981), pp. 185-203.

${ }^{7}$ Pratt, W.K., "Correlation techniques of image registration", IEEE Transactions on Aerospace Electronic Systems, vol. 10, pp. 353-358, May 1974.

${ }^{8}$ Jutamulia, S., "Joint transform correlators and their applications”, Proceedings SPIE, 1812 (1992), pp. $233-243$.

${ }^{9}$ Goodman, J.W., Introduction to Fourier optics. McGraw-Hill, New York 1968.

${ }^{10}$ Tchernykh, V., Dyblenko, S., Janschek, K., Seifart, K., Harnisch, B., “Airborne test results for a smart pushbroom imaging system with optoelectronic image correction", In: Sensors, Systems and Next-Generation Satellites VII, Proceedings of SPIE, Vol. 5234 (2004), pp.550-559.

11 Janschek, K., Tchernykh, V., Dyblenko, S., "Opto-Mechatronic Image Stabilization for a Compact Space Camera", Preprints of the 3rd IFAC Conference on Mechatronic Systems - Mechatronics 2004, 6-8 September 2004, Sydney, Australia, pp.547-552. Congress Best Paper Award.

${ }^{12}$ Tchernykh, V., Janschek, K., Dyblenko, S., "Space application of a self-calibrating optical processor or harsh mechanical environment”, Proceedings of 1st IFAC Conference on Mechatronic Systems - Mechatronics 2000, September 18-20, 2000, Darmstadt, Germany. Pergamon-Elsevier Science. vol. 3, (2000), pp.309-314.

13 Janschek, K., Tchernykh, V., Dyblenko, S., „Verfahren zur automatischen Korrektur von durch Verformungen hervorgerufenen Fehlern Optischer Korrelatoren und Selbstkorrigierender Optischer Korrelator vom Typ JTC“, Deutsches Patent Nr. 10047504 B4, Erteilt: 03.03.2005.

${ }^{14}$ Tchernykh, V., Beck, M., Janschek, K., “An Embedded Optical Flow Processor for Visual Navigation using Optical Correlator Technology", to appear in Proceedings of the IROS 2006 IEEE/RSJ International Conference on Intelligent Robots and Systems, 9-15 October 2006, Beijing, China, paper no. 1227. 